MATEC Web of Conferences 1, 08003 (2012)

DOI: $10.1051 /$ matecconf $/ 20120108003$

(C) Owned by the authors, published by EDP Sciences, 2012

\title{
Nonlinear dynamics and control strategies: On a energy harvester vibrating system with a linear form to non-ideal motor torque
}

\author{
I. Iliuk ${ }^{1}$, J. M. Balthazar ${ }^{2}$, A. M. Tusset ${ }^{3}$, J. L. P. Felix ${ }^{4}$ and B. R. de Pontes $\mathrm{Jr}^{1}$ \\ ${ }^{1}$ UNESP-São Paulo State University, Bauru, São Paulo, Brazil \\ ${ }^{2}$ UNESP-São Paulo State University, Rio Claro, São Paulo, Brazil \\ ${ }^{3}$ UTFPR- Paraná Federal Technological University, Ponta Grossa, Paraná, Brazil \\ ${ }^{4}$ UNIPAMPA-Pampa Federal University, Bagé, Rio Grande do Sul, Brazil
}

\begin{abstract}
In this paper, we deal with the research of a vibrating model of an energy harvester device, including the nonlinearities in the model of the piezoelectric coupling and the non-ideal excitation. We show, using numerical simulations, in the analysis of the dynamic responses, that the harvested power is influenced by non-linear vibrations of the structure. Chaotic behavior was also observed, causing of the loss of energy throughout the simulation time. Using a perturbation technique, we find an approximate analytical solution for the non-ideal system. Then, we apply both two control techniques, to keep the considered system, into a stable condition. Both the State Dependent Ricatti Equation (SDRE) control as the feedback control by changing the energy of the oscillator, were efficient in controlling of the considered non-ideal system.
\end{abstract}

\section{Introduction}

In the last years, we have been seeing a necessity of an energy source smaller than the usual and more efficient, for design the devices based on new technologies, so that the research on Energy Harvesting, has increasing substantially as an example, see [1]. In the process of Energy harvesting, the electrical energy is obtained through of conversion of mechanical energy, created by an environment vibration source through a type of transductor, for instance as a piezoceramic thin film, according to [2-3], among others authors. Because of the constitutive laws of piezoelectric materials, the role of nonlinearities, in the electromechanical coupling in the design of energy harvesting system, must be taken into account [4].

In previous works, we proposed a model of a nonideal energy harvesting, excited by a non-ideal energy source and with nonlinear piezoelectric coupling. Was investigated the dynamics of the system, the influence of the Sommerfeld effect over the system response, where a chaotic behavior was found and also high energy orbits inside the resonance region. The maximum power harvested was achieved to the model [5-7]. In this case, the energy source is said to be non ideal, in contrast to the ideal source whose amplitude and frequency are independent of the motion of the structure. For more details see [8]. In this paper, using a perturbation technique, we find an approximate analytical solution for the considered non-ideal system. Then, we applied two control techniques to keep the system in a stable condition. The SDRE method is a technique for nonlinear optimal control that synthesizes a feedback control law, which is a function of the state. The method linearizes the system around a point, so that you can use the same procedure of the method LQR (Linear Quadratic Regulator) [9]. Also we applied the feedback control method proposed by [10]. Where the method consists in suppressing of chaos by using a small-amplitude control signal, applied to alter the energy of a chaotic system, so as to steer its trajectory to a stable periodic orbit.

\section{Non-ideal energy harvester system (NIEH)}

In the figure 1 , we can see the schematic of the adopted model to the non-ideal system as defined in our previous works, for more details see [5-7]. Let's consider $M=m_{1}+m_{0}$ as the total mass of system, $x$ the vertical displacement of the beam, $c$ the mechanical damping, the nonlinear spring stiffness, given by $K=-k_{1} X-k_{2} X^{3}, \varphi$ is the rotation angle of the D.C. motor shaft, the parameters $r$ and $m_{0}$ are the eccentricity and mass of unbalanced shaft of the D.C. electric motor, $I=J+m_{0} r^{2}$ it is the moment of inertia of the rotor. The resistive torque applied to the motor is represented by function $H(\dot{\varphi})$ and the driving torque of the source of energy (motor) is represented by $L(\dot{\varphi})$ in [8]. In this work we consider a linear motor. A comparison between linear e exponential motor can be seen in [11]. We take $L(\dot{\varphi})$ as (linear) $L(\dot{\varphi})-H(\dot{\varphi})=V_{1}-V_{2} \dot{\varphi}$. Where $V_{1}$ is related to the voltage applied across to the armature of the D.C. motor (a control parameter) and $V_{2}$ is a constant that will be considered for each model of DC motor used. 


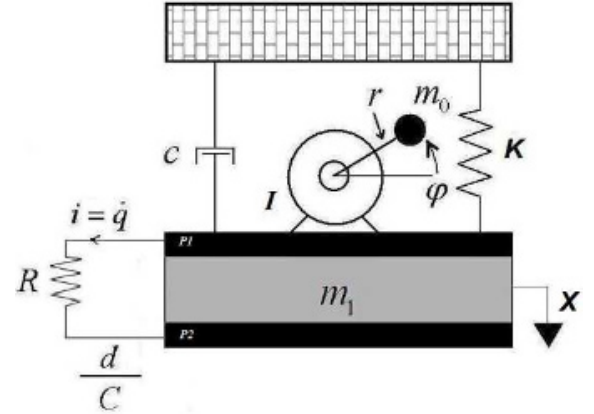

Fig. 1. Schematic model of (NIEH)

According to [4], the electrical charge developed in the coupled circuit, it is given by $q$ and the term $\frac{d(X)}{C} q$ represents the piezoelectric coupling to the mechanical component, with a strain-dependent coupling coefficient $d(X)$. The voltage $V$ across the piezoelectric material has the following form:

$V=-\frac{d(X)}{C} X+\frac{q}{C}$

Here the $C$ represents the piezoelectric capacitance and with $V=-R \dot{q}$.The (NIEH) coupled governing equations of motion are given by:

$$
\begin{aligned}
& M \ddot{X}+c \dot{X}+k_{1} X+k_{2} X^{3}=m_{0} r\left(\ddot{\varphi} \cos \varphi-\dot{\varphi}^{2} \sin \varphi\right)+\frac{d(X)}{C} q \\
& I \ddot{\varphi}=m_{0} r \ddot{X} \cos \varphi+L(\dot{\varphi})-H(\dot{\varphi}) \\
& R \dot{q}-\frac{d(X)}{C} X+\frac{q}{C}=0
\end{aligned}
$$

Next, we will consider the following non-dimensional parameters:

$\omega_{0}=\sqrt{k_{1} / M}, \tau=\omega_{0} t, x=\frac{X}{r}, v=\frac{q}{q_{0}}, \mu_{1}=\frac{V_{1}}{I \omega_{0}^{2}}, \mu_{2}=\frac{V_{2}}{I \omega_{0}}$,

$\gamma=\frac{m_{0} r^{2}}{I}, \rho \equiv R C \omega_{0}, \Theta \equiv r d_{n l i n}, \varepsilon \equiv\left(q_{0} / r\right)^{2} /(C k)$

$\alpha=\frac{c}{M \omega_{0}}, b=\frac{k_{2} r^{2}}{M \omega_{0}^{2}}, \delta_{1}=\frac{m_{0}}{M}, \delta_{2}=\frac{m_{0} \omega_{0}^{2}}{M}$,

$\hat{d}(x)=\theta(1+\Theta|x|), \hat{d}(x) \equiv\left(\frac{r}{q_{0}}\right) d(r), \theta \equiv\left(\frac{r}{q_{0}}\right) d_{l i n}$,

$\Gamma\left(\varphi^{\prime}\right)=\mu_{1}-\mu_{2} \dot{\varphi}$

The dimensionless piezoelectric coupling coefficient, used by us, was suggested by [4] as being $\hat{d}(x)=\theta(1+\Theta|x|)$, where the piezoelectric coefficient is represented by a linear part, represented by $\theta$ and nonlinear part defined by $\Theta$, so we may reduce the governing equations of motion to:

$x^{\prime \prime}+x=\varepsilon\left(\delta_{1} \varphi^{\prime \prime} \cos \varphi-\delta_{2} \varphi^{2} \sin \varphi-\alpha x^{\prime}-b x^{3}+\theta(1+\Theta|x|) v\right)$

$\varphi^{\prime \prime}=\varepsilon\left(\gamma x^{\prime \prime} \cos \varphi+\Gamma\left(\varphi^{\prime}\right)\right)$

$v^{\prime}=(\theta(1+\Theta|x|) x-v) / \rho$

Using of new variables: $x_{1}=x, x_{2}=x^{\prime}, x_{3}=\varphi$, $x_{4}=\varphi^{\prime}, x_{5}=v$, the equations in state space become:

$$
\begin{aligned}
& \dot{x}_{1}=x_{2} \\
& \dot{x}_{2}=\varepsilon\left(-\alpha x_{2}-b x_{1}^{3}+\theta\left(1+\Theta\left|x_{1}\right|\right) x_{5}-\delta_{1} \dot{x}_{4} \cos x_{3}+\delta_{2} x_{4}^{2} \sin x_{3}\right)-x_{1} \\
& \dot{x}_{3}=x_{4} \\
& \dot{x}_{4}=\varepsilon\left(\gamma \dot{x}_{2} \cos x_{3}+\Gamma\left(x_{4}\right)\right) \\
& \dot{x}_{5}=\left(\theta\left(1+\Theta\left|x_{1}\right|\right) x_{1}-x_{5}\right) / \rho
\end{aligned}
$$

The power harvested from the mechanical component is $V^{2} / R$, so the non-dimensional electrical power harvested from the system, was identified by [4] as being: $P=\rho v^{2}$

\section{Numerical simulations results}

We used the following values for the parameters in the numerical simulations, those were carried out:

$$
\begin{aligned}
& \alpha=0.01, b=0.25, \rho=1.0, \varepsilon=0.10, \delta_{1}=0.40, \delta_{2}=0.40, \\
& \gamma=0.60, \mu_{1}=2.50, \mu_{2}=1.5, \theta=0.01, \Theta=0.50, x_{1}(0)=0, \\
& x_{2}(0)=0, x_{3}(0)=0, x_{4}(0)=0, x_{5}(0)=0 ;
\end{aligned}
$$

In figure 2 we have the dynamic response of the (NIEH). Through the analysis of the phase portrait and history of displacement in time, it may be noted that chaotic behavior occurs in the system, it can be attributed to the interaction between the non-ideal energy source and the structure. Note that in figure 2-d the presence of a positive Lyapunov exponent. In this condition the system is unstable, so that maximum power harvested tends to decrease along the time.
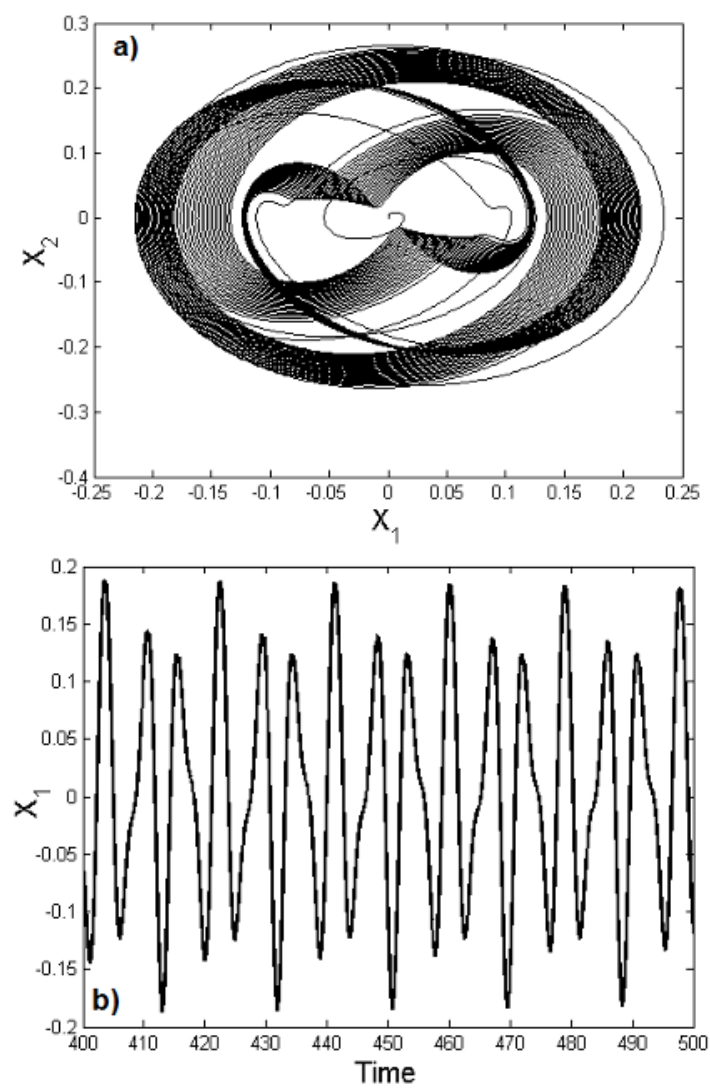

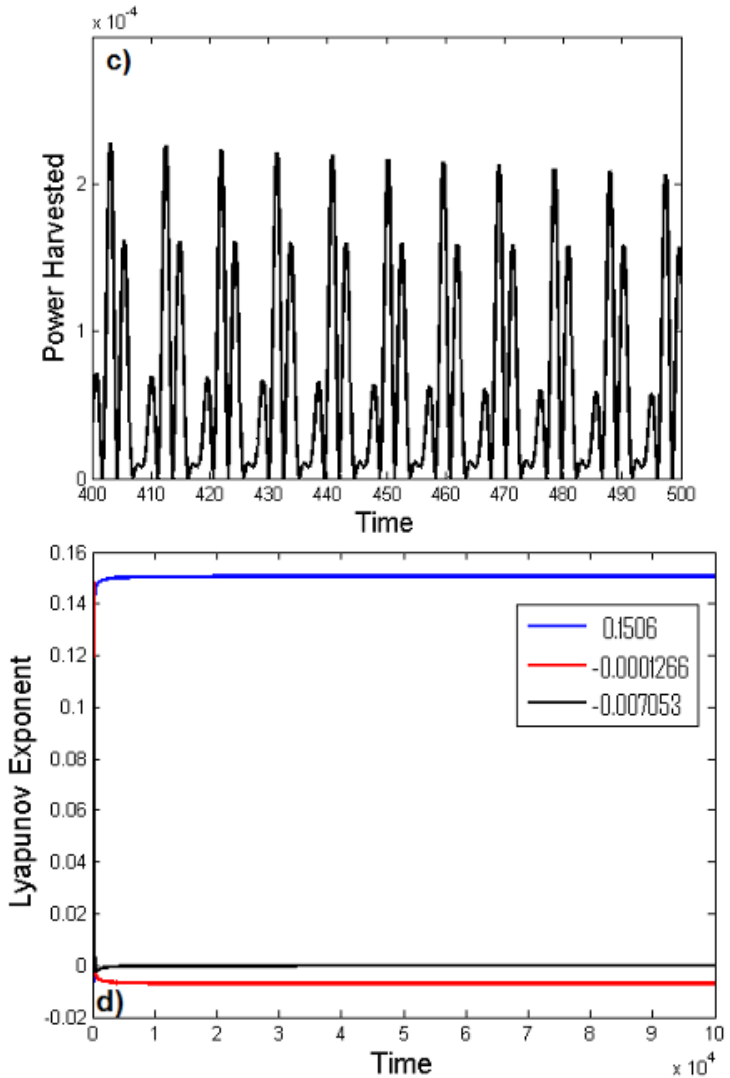

Fig. 2. Dynamic of the (NIEH) (a) Phase portrait;

(b) History of displacement in time; (c) Maximum power harvested; (d) Largest Lyapunov exponent

\section{Searching of an approximate analytic solution}

We will find an approximate analytical solution for the energy harvesting system, using the averaging method, seeking of an approximate solution, which must be uniformly valid for a small parameter $\mathcal{E}$ (As explained in detail in [12]). To apply the averaging method, in the problem non-ideal, it is considered that the damping term, must appear in same order which the nonlinearities and excitation. We also consider the case of primary resonance, where the average angular velocity of the DC motor, varies close to the natural frequency of the system, i.e., $\omega_{0} \approx \varphi^{\prime}$. From the system (6), taking the equations dependent of the small parameter, we will seek a solution with the form:

$x=a \cos (\varphi+\beta)$;

Introducing of the detuning parameter $\Delta$, as follow:

$\varphi^{\prime}=1+\Delta$

Using of the method of variation of parameters, we have that:

$a^{\prime} \cos (\varphi+\beta)-a\left(\Delta+\beta^{\prime}\right) \sin (\varphi+\beta)=0$

So that:

$x^{\prime}=-a \sin (\varphi+\beta)$

And:

$x^{\prime \prime}=-a^{\prime} \sin (\varphi+\beta)-a\left(1+\Delta+\beta^{\prime}\right) \cos (\varphi+\beta)$
Substituting of (9), (10), (12) and (13) into (6) it leads to: $-a^{\prime} \sin (\varphi+\beta)-a\left(\Delta+\beta^{\prime}\right) \cos (\varphi+\beta)=$ $\varepsilon\left(\delta_{1} \Delta^{\prime} \cos \varphi-\delta_{2}(1+\Delta)^{2} \sin \varphi+\alpha a \sin (\varphi+\beta)-\right.$ $\left.b a^{3} \cos ^{3}(\varphi+\beta)+\theta(1+\Theta|a \cos (\varphi+\beta)|) v\right) \sin (\varphi+\beta)$

$\Delta^{\prime}=\varepsilon\left(-\gamma\left[a^{\prime} \sin (\varphi+\beta)+a\left(1+\Delta+\beta^{\prime}\right) \cos (\varphi+\beta)\right] \cos \varphi+\Gamma(1+\Delta)\right)$

Solving (9) and (14) for $a^{\prime}$ and $\beta^{\prime}$ leads to:

$a^{\prime}=-\varepsilon\left[\delta_{1} \Delta^{\prime} \cos \varphi-\delta_{2}(1+\Delta)^{2} \sin \varphi+\alpha a \sin (\varphi+\beta)-\right.$

$\left.b a^{3} \cos ^{3}(\varphi+\beta)+\theta(1+\Theta|a \cos (\varphi+\beta)|) v\right] \sin (\varphi+\beta)$

$\beta^{\prime}=-\Delta-\varepsilon / a^{[}\left[\delta_{1} \Delta^{\prime} \cos \varphi-\delta_{2}(1+\Delta)^{2} \sin \varphi+\alpha a \sin (\varphi+\beta)-\right.$

$\left.b a^{3} \cos ^{3}(\varphi+\beta)+\theta(1+\Theta|a \cos (\varphi+\beta)|) v\right] \cos (\varphi+\beta)$

The equations (15) to (17) are equivalent to the system (6). Now rewrite:

$\Delta=\varepsilon \sigma$;

And as a first simplification neglects all terms $O\left(\varepsilon^{2}\right)$, which appear (15) to (17) and obtain:

$a^{\prime}=\varepsilon\left[\delta_{2} \sin \varphi-\alpha a \sin (\varphi+\beta)+b a^{3} \cos ^{3}(\varphi+\beta)-\right.$

$\theta(1+\Theta|a \cos (\varphi+\beta)|) v] \sin (\varphi+\beta)$

$\beta=-\varepsilon\left[\sigma+1 / a\left(-\delta_{2} \sin \varphi+\alpha a \sin (\varphi+\beta)-b a^{3} \cos ^{3}(\varphi+\beta)+\right.\right.$

$\theta(1+\Theta|a \cos (\varphi+\beta)|) v) \cos (\varphi+\beta)]$

$\Delta^{\prime}=\varepsilon[\Gamma-\gamma a \cos (\varphi+\beta) \cos \varphi]$

As a second simplification, it can be considered that

$a, \quad \beta$ and $\sigma$ are constants over one cycle and integrating (averaging) the equations on a cycle will be:

$$
\begin{aligned}
& a^{\prime}=\varepsilon\left[1 / 2 \delta_{2} \cos \beta-1 / 2 \alpha a\right] \\
& \beta^{\prime}=-\varepsilon\left[\sigma+1 / 2 \frac{\delta_{2} \sin \beta}{a}-3 / 8 a^{2} b\right] \\
& \Delta^{\prime}=\varepsilon[\Gamma-1 / 2 \gamma a \cos \beta]
\end{aligned}
$$

Now solving the equations, numerically, the solution is:

$\mathrm{X}^{*}=-0.2007560184 * \cos (\tau+1.575815248)$

Below, the periodic solution is depicted in figure 3 .

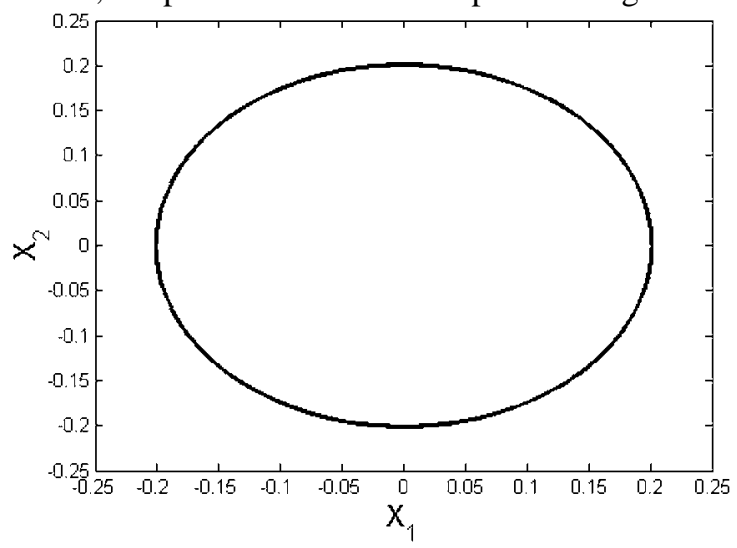

Fig. 3. Averaging Method - Periodic solution obtained 


\section{Application of the SDRE control method}

The objective is to find the control, such that the response of the controlled system, results in the asymptotically stable periodic orbit, by using the feedback control. The control proposed in this paper, uses the SDRE control [9]. Introducing the control in the considered system, it has the following system:

$\dot{x}=A(x) x+F+B u$

Where: $u$ it the feedback control, obtained from: $u=$ $-\mathrm{R}^{-1} B^{T} e=-\mathrm{K}\left(x-x^{*}\right)$, where $x$ represents the states, and $x^{*}$ is the desired state. $\mathrm{K}=\operatorname{lq} r(A(x), B, Q, \mathrm{R})$ is obtained through the command of Matlab ${ }^{\circledR}$. Since the objective is to control the states $x_{1}$ and $x_{2}$ will be implemented in the controlled system:

$\dot{x}_{1}=x_{2}$

$\dot{x}_{2}=\frac{1}{\Delta}\left[-\varepsilon \alpha x_{2}-x_{1}-\varepsilon b x_{1}^{3}+\varepsilon\left(\theta x_{5}+\theta \Theta\left|x_{1}\right| x_{5}\right)\right.$

$\left.-\varepsilon\left(\delta_{2} x_{4}^{2} \sin x_{3}\right)+\varepsilon^{2}\left(\delta_{1} \cos x_{3}\right)\left(\mu_{1}-\mu_{2} x_{4}\right)\right]$

$\dot{x}_{3}=x_{4}$

$\dot{x}_{4}=\frac{1}{\Delta}\left[\varepsilon\left(\mu_{1}-\mu_{2} x_{4}\right)+\varepsilon \gamma \cos x_{3}\right.$

$\left.\left(-\varepsilon \alpha x_{2}-x_{1}-\varepsilon b x_{1}^{3}+\varepsilon\left(\theta x_{5}+\theta \Theta\left|x_{1}\right| x_{5}\right)-\varepsilon \delta_{2} x_{4}^{2} \sin x_{3}\right)\right]$

$\dot{x}_{5}=\left(\left(\theta x_{1}+\theta \Theta\left|x_{1}\right| x_{1}\right)-x_{5}\right) / \rho$

Where: $\Delta=1-\varepsilon^{2} \delta_{1} \gamma \cos ^{2} x_{3}$

Other states will be considered as a disturbance. Considering the system (27), in the form (26), we'll have:

$\dot{x}=\left[\begin{array}{cc}0 & 1 \\ \frac{-\varepsilon b x_{1}^{2}}{\Delta} & \frac{-\varepsilon \alpha}{\Delta}\end{array}\right] x+$

$\left[\frac{\varepsilon}{\Delta}\left[\left(\theta x_{5}+\theta \Theta\left|x_{1}\right| x_{5}\right)-\left(\delta_{2} x_{4}^{2} \sin x_{3}\right)+\mathcal{E}\left(\delta_{1} \cos x_{3}\right)\left(\mu_{1}-\mu_{2} x_{4}\right)\right]\right]+\left[\begin{array}{l}0 \\ 1\end{array}\right] u$

Where:

$$
\begin{aligned}
A & =\left[\begin{array}{cc}
0 & 1 \\
\frac{-\varepsilon b x_{1}^{2}}{\Delta} & \frac{-\varepsilon \alpha}{\Delta}
\end{array}\right], B=\left[\begin{array}{l}
0 \\
1
\end{array}\right] \text { and } \\
F & =\left[\frac{\varepsilon}{\Delta}\left[\left(\theta x_{5}+\theta \Theta\left|x_{1}\right| x_{5}\right)-\left(\delta_{2} x_{4}^{2} \sin x_{3}\right)+\varepsilon\left(\delta_{1} \cos x_{3}\right)\left(\mu_{1}-\mu_{2} x_{4}\right)\right]\right]
\end{aligned}
$$

Defining the matrices $Q=10^{5} I_{2 X_{2}}$ and $\mathrm{R}=10^{-3} I_{1 X 1}$, we will obtain the control: $u=-\mathrm{Ke}$. Since, $x_{3}, x_{4}$ and $x_{5}$ are obtained from (28). Figure 4 shows the system controlled with the control $u$ for the desired state (26).
Here, we can be observed the efficiency of the proposed control system: to bring the chaotic behavior to the desired orbit (25) (here is considered the application of the control (26) in (7)).
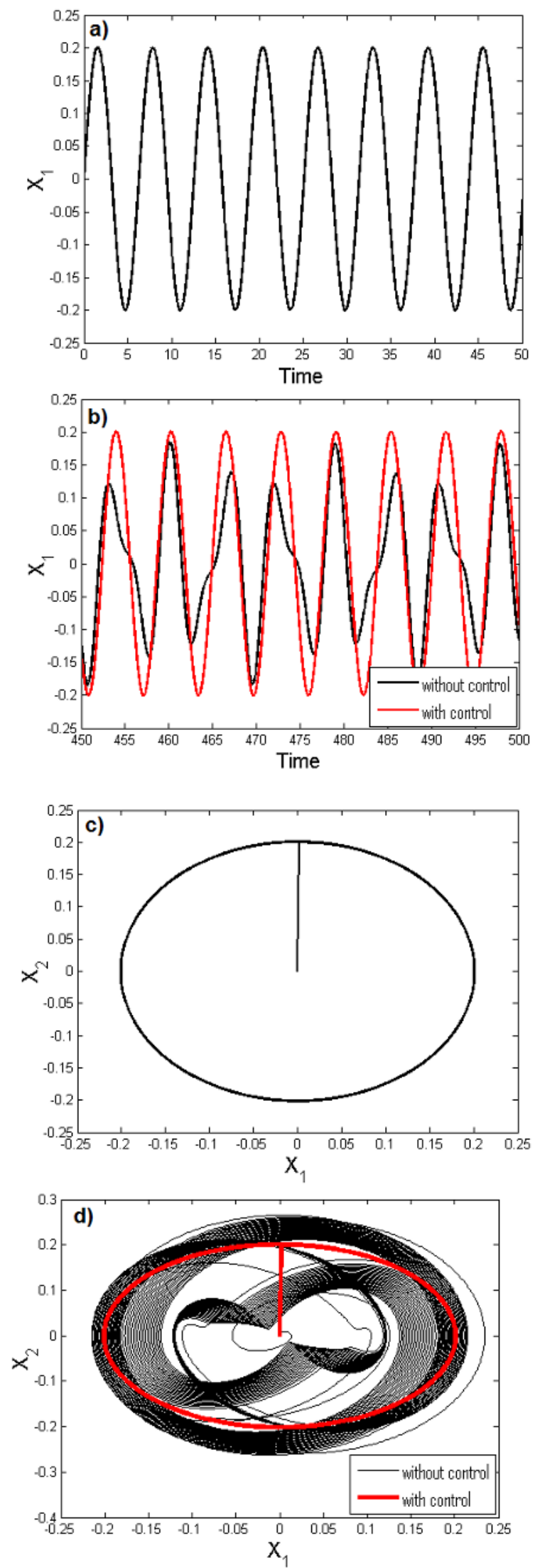

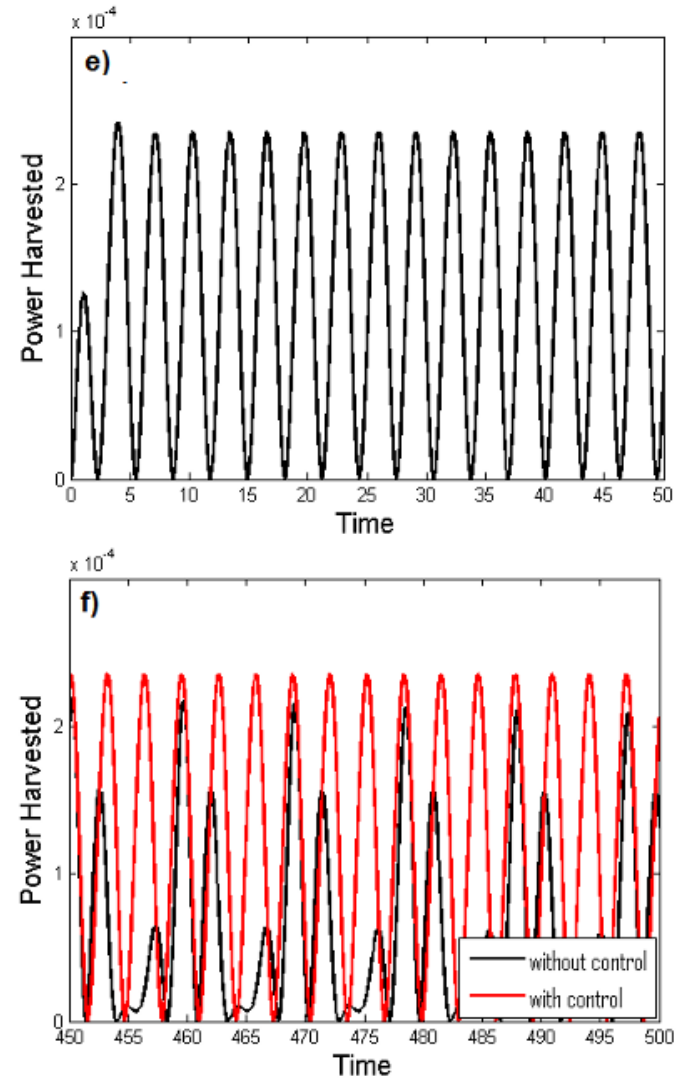

Fig. 4. SDRE control strategy: (a), (c) and (e) represents the solution obtained by control strategy; in (b), (d) and (f) a comparison between controlled and uncontrolled system; (b) History of displacement in time; (d) Phase portrait; (e) Power harvested

\section{Feedback altering of the vibrating energy}

The objective is to find the control, such that the response of the controlled system, results in the asymptotically stable periodic orbit, by using a control proposed by [10]. Introducing of the control in system, it has the following system:

$\dot{x}_{1}=x_{2}$

$\dot{x}_{2}=\varepsilon\left(-\alpha x_{2}-b x_{1}^{3}+\theta\left(1+\Theta\left|x_{1}\right|\right) x_{5}-\delta_{1} \dot{x}_{4} \cos x_{3}+\delta_{2} x_{4}^{2} \sin x_{3}\right)-x_{1}+u$

$\dot{x}_{3}=x_{4}$

$\dot{x}_{4}=\varepsilon\left(\gamma \dot{x}_{2} \cos x_{3}+\Gamma\left(x_{4}\right)\right)$

$\dot{x}_{5}=\left(\theta\left(1+\Theta\left|x_{1}\right|\right) x_{1}-x_{5}\right) / \rho$

Where: $u$ feedback alters the oscillation energy it the feedback control, obtained from:

$u=-k \tanh (\chi \dot{x})$

Taking in account the values to $\kappa=0.4$ and $\chi=0.5$, the figure 5 shows the system response excited by the linear function torque, when is applied to the control technique proposed by [10]. Note that the control was effective, in keeping the system stable one periodic solution.
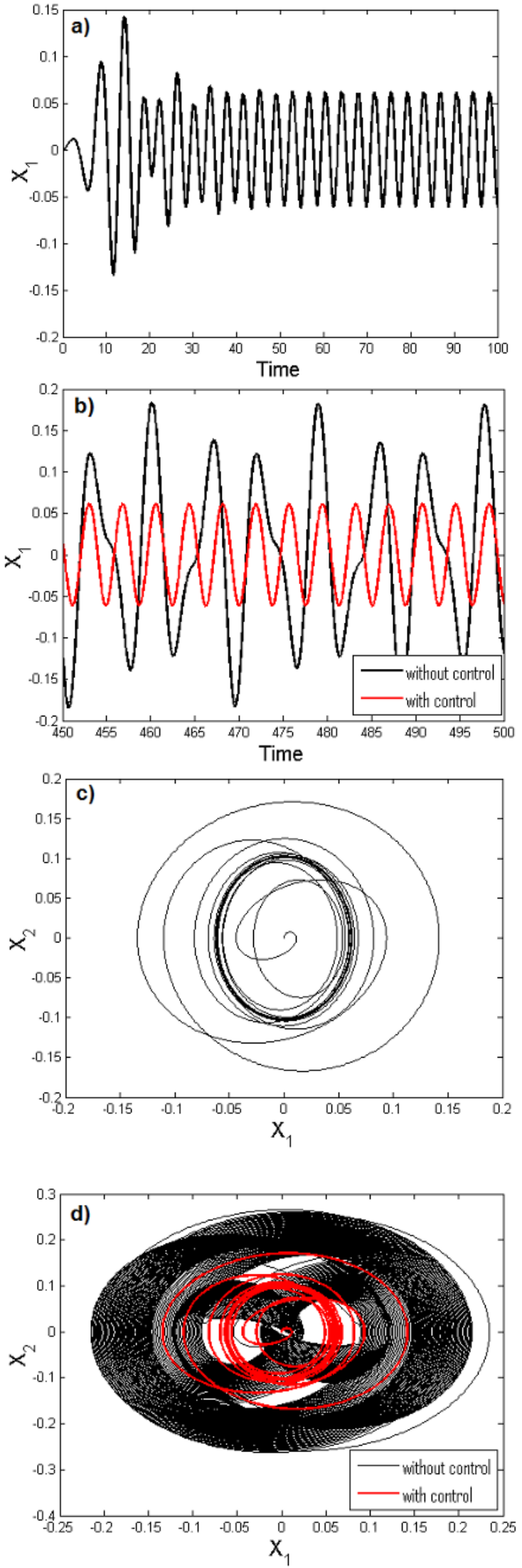

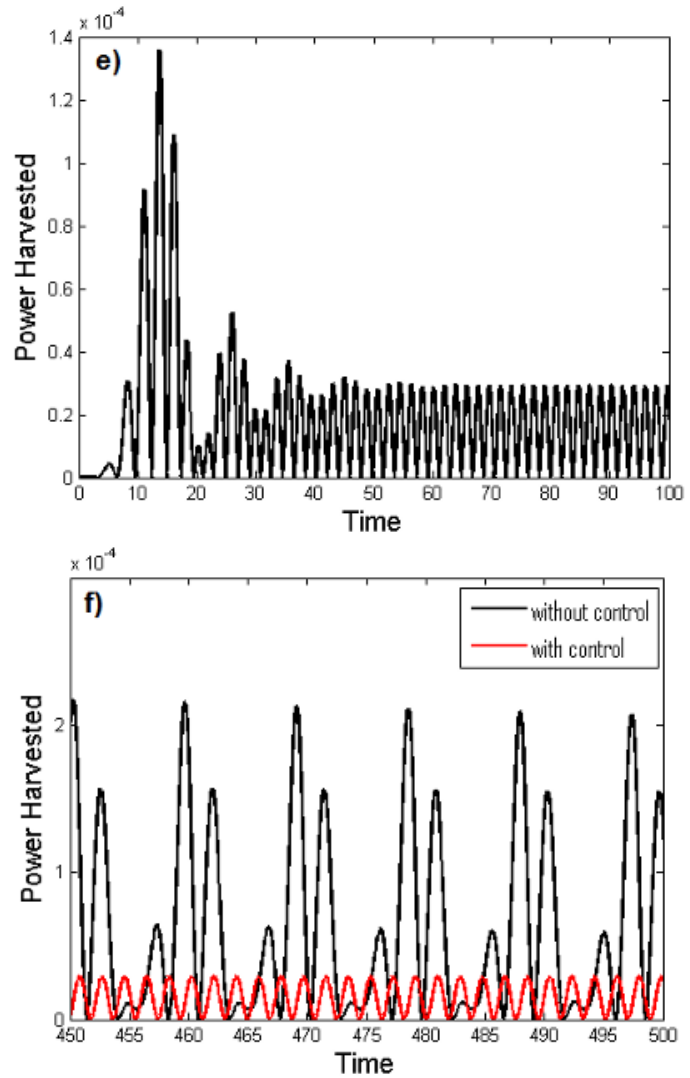

Fig. 5. Feedback control strategy: (a), (c) and (e) represents the solution obtained by control strategy; in (b), (d) and (f) a comparison between controlled and uncontrolled system; (b) History of displacement in time; (d) Phase portrait; (e) Power harvested

\section{Conclusions}

The maintenance of the considered vibrating system in a stable periodic orbit is of paramount importance to harvest energy from the electromechanical device. Using a perturbation technique, we can find an approximate analytical solution for the non-ideal system where we can seek the solution that maximizes the system's response.

The averaging method was applied with success to solve the proposed problem. We used the SDRE control to bring and maintain this chaotic system periodic solution obtaining by the application of the averaging method.

The answers were satisfactory, because the energy harvested was stable, validating of the solution, which was obtained by this technique de control. The advantage of the using the control as studied by [10], is that it does not require find an analytical solution initially.

But the solution did not get a great harvest of energy, due to reduced vibration amplitude. Both of control techniques applied in the model of non-ideal energy harvester proposed in this paper, could prove effective, while keeping the system stable.

The main advantage of using a control technique to stabilize the system is the reducing of the need for complex filters rectifiers in the output from energy harvesting system.

\section{Acknowledgments}

The authors thank FAPESP, CNPq and CAPES, Brazilian financial agencies.

\section{References}

1. S. Priya, D.J. Inman, Energy Harvesting Technologies (Springer Science Business Media, LLC, 2009)

2. H.A. Sodano, D.J. Inman, G. Park, A Review of Power Harvesting from Vibration Using Piezoelectric Materials, Shock Vib. Dig., 36, 197205 (2004)

3. S.R. Anton, H.A. Sodano, A Review of Power Harvesting Using Piezoelectric Materials (20032006), Smart Mater. Struct., 16 (2007)

4. A. Triplett, D. D. Quinn, The Effect of Non-linear Piezoelectric Coupling on Vibration-based Energy Harvesting, Journal of Intelligent Material Systems and Structures, 20, 1959-1967 (2009)

5. I. Iliuk, J. M. Balthazar, A. M. Tusset, J. L. P. Felix, B. R. de Pontes Jr, On a vibrating model of energy harvester, with nonlinear piezoelectric coupling and excited by a non-ideal motor, $11^{\text {th }}$ conference on dynamical systems theory and applications, Lodz, Poland, (2011).

6. I. Iliuk, J. M. Balthazar, A. M. Tusset, J. L. P. Felix, B. R. de Pontes Jr, On a Mathematical Model of the Non-Ideal Energy Harvester Vibrating System, Proceedings of the $21^{\text {st }}$ International Congress of Mechanical Engineering, ISSN 2176-5480, Natal, RN, Brazil, (2011).

7. I. Iliuk, J. M. Balthazar, A. M. Tusset, J. L. P. Felix, B. R. de Pontes Jr, Sommerfeld Effect in a Vibration Model of Non-Linear Energy Harvester Excited by a Non-Ideal Energy Source, DINCON, 10 Conferência Brasileira de Dinâmica, Controle e Aplicações, Aguas de Lindóia, SP, Brasil, (2011).

8. J.M. Balthazar, D.T. Mook, H. I .Weber, R. M.L.R.F. Brasil, A. Fenili, D. Belato and J.L.P. Felix, An Overview On Non-ideal Vibrations, Meccanica, 38, 613-621, (2003).

9. A. Fenili, J.M. Balthazar, The rigid-flexible nonlinear robotic manipulator, Modeling and control, Communications in Nonlinear Science and Numerical Simulation, 16, Issue 5, 2332-2341 (2011)

10. V. Tereshko, R. Chacon, V. Preciado, Controlling chaotic oscillators by altering their energy, Physics Letters A, 320, 408-416 (2004)

11. I. Iliuk, J. M. Balthazar, A. M. Tusset, J. L. P. Felix, B. R. de Pontes Jr, On a Vibrating Model of Energy Harvester, with Nonlinear Piezoelectric Coupling and Excited by a Non-Ideal Motor, submitted,(2011)

12. A. H. Nayfeh, D. T. Mook, Nonlinear Oscillations. Wiley, (New York, 1979) 